\title{
Qualidade de Serviço: um estudo bibliométrico nas BASES DE DADOS INTERNACIONAIS
}

\author{
Quality of Service: a study in databases bibliometric international
}

\author{
Deosir Flávio Lobo de Castro Junior \\ Professor do Instituto Federal Catarinense (IFSC) - Florianópolis - SC - Brasil. E-mail: deosir@ifsc.edu.br \\ Elvis Silveira-Martins \\ Professor Faculdade de Administração e de Turismo (FAT) Universidade Federal de Pelotas (UFPel) - Pelotas - RS - Brasil. E-mail: \\ elvis.professor@gmail.com
}

Marcelo Augusto Menezes Deluca

Professor do CAD/UFSC - Marketing e Pesquisa de Marketing - Florianópolis - SC - Brasil. E-mail: marcelo.deluca@ufsc.br

Carlos Ricardo Rossetto

Professor da Universidade do Vale do Itajaí (UNIVALI) - Biguaçu- SC - Brasil. E-mail: rossetto@univali.br

\section{Resumo}

O objetivo deste artigo é servir de fonte de referência sobre Qualidade de Serviços para futuras pesquisas. Após levantamento nas bases de dados internacionais, EBSCO e ProQuest, serão apresentados os resultados sobre o estado da arte desse assunto. O método utilizado foi a bibliometria, por isso, foram investigados 132 artigos de um universo de 13.427. Os trabalhos analisados compreendem o período de 1985 a 2011. Dentre as contribuições, os resultados e as conclusões para futuras pesquisas são apresentados: i) os autores mais citados; ii) a metodologia, as dimensões e o questionário mais utilizado; iii) as publicações mais referenciadas; iv) os jornais internacionais com mais publicações sobre o tema; v) a distribuição da quantidade de publicações por ano; vi) as redes de autores; vii) a rede de instituições de ensino; viii) os termos utilizados na busca em bases internacionais; ix) as relações estudadas nos 132 artigos; $\mathrm{x}$ ) os critérios para escolha da metodologia na pesquisa em qualidade de serviços; xi) o paradigma mais utilizado; e xii) as 160 referências de alto impacto.

Palavras-chave: Qualidade de Serviços. Serviços. Bibliometria.

\section{Abstract}

The purpose of this article is to serve as a source of references on Quality of Service for future research. After surveying the international databases, EBSCO and ProQuest, the results on the state of the art in this issue are presented. The method used was the bibliometrics, and 132 items from a universe of 13,427 were investigated. The analyzed works cover the period from 1985 to 2011. Among the contributions, results and conclusions for future research are presented: i) most cited authors ii) most used methodology, dimensions and questionnaire; iii) most referenced publications iv) international journals with most publications on the subject, $v$ ) distribution of the number of publications per year; vi) authors networks vii) educational institutions network; viii) terms used in the search in international databases; ix) the relationships studied in 132 articles; $\mathrm{x})$ criteria for choice of methodology in the research on quality of services; xi) most often used paradigm, and xii) 160 high impact references.

Key words: Quality of Service. Service. Bibliometrics. 


\section{INTRODUÇÃo}

No setor de serviços existe uma diversidade de empresas dos mais variados tamanhos e segmentos que são submetidas diariamente ao dinamismo do mercado, fruto de acirrada concorrência que acaba por forçar os gestores a tomarem decisões em tempo real para atender as exigências de seus consumidores e usuários. Estas características direcionaram, já na década de 1980, alguns pesquisadores a investigar a temática qualidade de serviços, concluindo que, a percepção dos gestores é, por muitas vezes, diferente daquela de seus consumidores. Ao existir esta dificuldade, de manter uma visão realista da qualidade nos serviços prestados, outros estudos apontaram para a necessidade de conhecer as percepções dos clientes externos buscando contrastar com as dos gestores $e$ funcionários. (LOVELOCK, 1983; NIGHTINGALE, 1985; CALLAN, 1988; ARMISTEAD, 1989)

Willborn (1986), Collier (1987) e Rhyne (1988) estudaram a qualidade e concluíram que a garantia da qualidade de uma empresa serviço é tarefa de seus gestores. Edvardsson, Mattsson (1993) e Pitt e Jeantrout (1994), tomando a perspectiva de encontro de pessoas na prestação de serviços, mostram que os gestores recebem uma nova função para dar o apoio ao processo nesta interação. Pitt, Watson e Kavan (1995) argumentam que ignorando medidas de expectativas dos clientes, diminui-se o conhecimento dos gestores.

Diante desses aspectos, observa-se a importância de se discutir e entender qual o estado da arte sobre esse tema, que desperta o interesse de pesquisadores de marketing pelo mundo. Nessa perspectiva, o presente estudo objetiva bibliografar o tema qualidade de serviços nas bases de dados internacionais: $\mathrm{EBSCO} e$ ProQuest.

Com o objetivo de servir como fonte de referências para pesquisas futuras sobre o tema Qualidade de Serviços, foi realizada a pesquisa bibliométrica nas bases de dados internacionais EBSCO e ProQuest apresentando os resultados sobre o estado da arte deste assunto $e$ as características dos trabalhos científicos já publicados. Justifica-se o presente estudo pela necessidade de explorar a temática qualidade de serviços em produções científicas reconhecidas pela academia, verificando suas características e o estado da arte internacional, subsidiando assim novas pesquisas.
Outro aspecto que legitima esta pesquisa é o fato de não terem sido encontrados estudos similares com esta abordagem no Brasil.

Nesse sentido, este artigo está organizado em cinco seções. A primeira parte explora passo a passo a gênese, o desenvolvimento $e$ a consolidação da temática qualidade de serviços. Na segunda seção, apresenta-se a revisão da literatura que procura situar o contexto intelectual onde se empreendeu o estudo, na sequência, aborda-se a metodologia que orientou a condução do estudo sob a análise. Na quarta seção, discute-se e pondera-se sobre a análise dos dados bibliográficos e na última seção, tecem-se reflexões sobre os resultados e conclusões, além de novas direções e possibilidades para estudos sobre qualidade de serviços.

\section{Revisão de Literatura: Qualidade de SERVIÇO}

Lovelock (1983) e Bitner e Obermiller (1985) conceituam o serviços como sendo, qualquer ato ou desempenho que busque a satisfação do consumidor. Diferentemente do conceito de serviços, a definição da qualidade de serviços é mais complexa.

$\mathrm{O}$ conceito de qualidade de serviços tem sido construído sob diversos ângulos. A busca pela entrega da satisfação do consumidor é visto por Grönroos (1982), que a qualidade de serviços diferenciando-a entre a qualidade técnica (o que se recebe) e a funcional (o como se recebe), Parasuraman, Zeithaml e Berry (1985) comparando o serviço que esperam com as percepções do serviço que recebem; Cronin (1992) a avaliação de qualidade é realizado aferindo o desempenho da prestação de serviço. Apesar de não existir até o presente momento uma unanimidade, o paradigma da desconfirmação que é resultante da expectativa do serviço menos o que é percebido, é o paradigma mais utilizado em pesquisas.

Uma vez que a metodologia mais utilizada nas pesquisas é o SERVQUAL de Parasuraman, Zeithaml e Berry (1988, 1991, 1994), as dimensões da qualidade de serviço mais utilizadas são: Confiança, Atenção, Segurança, Empatia e Tangibilidade.

As dimensões servem para a entrega da qualidade dos serviços e para atender às quatro características 
específicas do serviço, como inseparabilidade, intangibilidade, perecibilidade e heterogeneidade. A seguir no Quadro 1 são apresentadas as questões utilizadas no SERVQUAL (1988, 1991, 1994).

As questões do instrumento de pesquisa utilizado pelo SERVQUAL de Parasuraman, Zeithaml e
Berry $(1988,1991,1994)$ também foram utilizadas por Cronin e Taylor $(1992,1994)$ mudando apenas o paradigma. Enquanto Parasuraman, Zeithaml e Berry (1988, 1991, 1994), utilizam o paradigma da desconfirmação (expectativa menos a percepção), Cronin e Taylor utilizam diretamente o desempenho.

\begin{tabular}{|c|c|}
\hline DiMENSÕES & EXPECTATIVAS SOBRE AS VARIÁvEIS \\
\hline \multirow{4}{*}{ 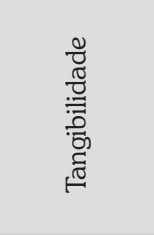 } & E1. Empresas excelentes terão equipamento com aparência moderna. \\
\hline & E2. As instalações das empresas excelentes são bonitas. \\
\hline & E3. Os funcionários de empresas excelentes terão boa aparência. \\
\hline & E4. Os materiais associados ao serviço terão aparência bem cuidada em uma empresa excelente. \\
\hline \multirow{5}{*}{ 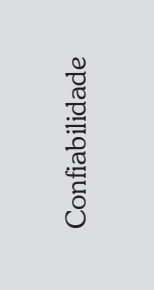 } & E5. Quando empresas excelentes prometem fazer alguma coisa por um determinado período, fazem. \\
\hline & E6. Quando os clientes têm um problema, as empresas excelentes demonstrarão interesse em resolvê-lo. \\
\hline & E7. Empresas excelentes prestarão o serviço de maneira certa na primeira vez. \\
\hline & E8. Empresas excelentes prestarão seus serviços no momento em que prometerem fazê-lo. \\
\hline & E9. Empresas excelentes insistirão em registros sem erros. \\
\hline \multirow{4}{*}{ 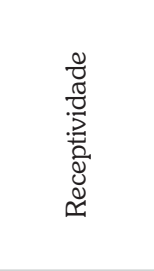 } & E10. Os funcionários de empresas excelentes dirão aos clientes exatamente quando o serviço será prestado. \\
\hline & E11. Os funcionários de empresas excelentes prestarão serviço imediato aos clientes. \\
\hline & E12. Os funcionários de empresas excelentes estarão dispostos a ajudar clientes. \\
\hline & $\begin{array}{l}\text { E13. Os funcionários de empresas excelentes nunca estarão ocupados demais para responder aos pedidos dos } \\
\text { clientes. }\end{array}$ \\
\hline \multirow{4}{*}{ 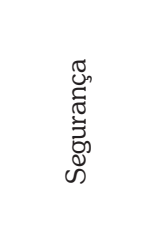 } & E14. O comportamento dos funcionários de empresas excelentes inspirará confiança nos clientes. \\
\hline & E15. Os funcionários de empresas excelentes se sentirão seguros em suas transações. \\
\hline & E16. Os funcionários de empresas excelentes serão corteses com os clientes. \\
\hline & E17. Os funcionários de empresas excelentes terão conhecimento para responder a perguntas dos clientes. \\
\hline \multirow{5}{*}{ 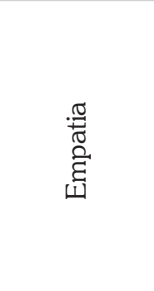 } & E18. Empresas excelentes darão atenção individual aos clientes. \\
\hline & E19. Empresas excelentes terão horário de funcionamento conveniente para todos os clientes. \\
\hline & E20. Empresas excelentes terão funcionários que dão atenção pessoal aos clientes. \\
\hline & E21. Empresas excelentes terão como prioridade os interesses do cliente. \\
\hline & E22. Os funcionários de empresas excelentes entenderão as necessidades específicas de seus clientes. \\
\hline
\end{tabular}

Quadro 1: Dimensão e indicadores - SERVQUAL (1988, 1991, 1994)

Fonte: Parasuraman, Zeithaml e Berry (1988)

Hamer (2003), objetivando dirimir as dúvidas dos pesquisadores por qual método utilizar, apresenta a contribuição sobre a escolha da metodologia apropriada a cada pesquisa, ao verificar os possíveis critérios de pesquisa como: precisão; valor diagnóstico; parcimônia; precisão e valor diagnóstico; precisão e parcimônia; $e$ valor diagnóstico e parcimônia. No Quadro 2 pode-se verificar o critério e a metodologia apropriada. 


\begin{tabular}{|l|l|}
\hline $\begin{array}{c}\text { O PRINCIPAL CRITÉRIO É } \\
\text { (CRITÉRIOS SÃo)... }\end{array}$ & O MÉTODO MAIS APROPRIADO É... \\
\hline Precisão & $\begin{array}{l}\text { Medição direta da Percepção da } \\
\text { Qualidade }\end{array}$ \\
\hline Valor Diagnóstico & $\begin{array}{l}\text { Medição separada das } \\
\text { Expectativas e do desempenho }\end{array}$ \\
\hline Parcimônia & $\begin{array}{l}\text { Medição direta da Percepção da } \\
\text { Qualidade }\end{array}$ \\
\hline Precisão e Valor \\
Diagnóstico & $\begin{array}{l}\text { Medição separada das } \\
\text { Expectativas e do desempenho }\end{array}$ \\
\hline Precisão e parcimônia & $\begin{array}{l}\text { Medição direta da Percepção da } \\
\text { Qualidade }\end{array}$ \\
\hline $\begin{array}{l}\text { Valor diagnóstico e } \\
\text { Parcimônia }\end{array}$ & Medição direta da desconfirmação \\
\hline
\end{tabular}

Quadro 2: Recomendações da medição de serviços e métodos de qualidade

Fonte: Hamer (2003, p. 41)

\section{Metodologia}

Os procedimentos metodológicos desta pesquisa estão alicerçados na técnica da bibliometria. De acordo com Araujo et al. (2000), esse tipo de estudo busca observar a evolução da literatura e o conhecimento produzido no decorrer dos anos.

Nesses moldes, foram utilizados os termos service quality, marketing strategy, marketing service e service encounter para localizar artigos referentes a qualidade de serviços nas bases de dados EBSCO e ProQuest. Considerou-se que essas palavras-chave deveriam estar contidas no título ou no resumo dos trabalhos. Foram desconsiderados todos os artigos que não se encontravam completos, além das críticas literárias. Do universo de 13.427 artigos, foram considerados apenas os artigos que possuíam Index $\mathrm{H}$ igual ou maior do que 5. Após esta seleção restaram 211 artigos da base EBSCO e 110 artigos originários da base ProQuest. O terceiro filtro aplicado foi o de exclusão de artigos que se encontravam nas duas bases e os que utilizavam os termos service quality, marketing strategy, marketing service e service encounter de maneira genérica, sem vínculo com a temática de qualidade de serviços.
Dessa forma, a seleção final dos artigos resultou em um total de 132 artigos válidos, sendo 121 artigos da base EBSCO e 11 artigos da base ProQuest.

Após a apresentação dos aspectos metodológicos que nortearam o desenvolvimento desta pesquisa, a seguir será realizada a análise bibliométrica sobre as publicações identificadas na coleta dos dados.

\section{Análise Bibliométrica}

Inicialmente foi investigado qual o autor mais citado nos estudos sobre serviço. Com esse critério, Parasuraman aparece como o mais citado com 255 referências, em seguida a autora Zeithaml com 125 citações, em terceiro Grönroos com 97 citações, em quarto Cronin com 85 citações e em quinto aparece Berry com 66 citações. No Quadro 4 são apresentadas as referências dos estudos destes pesquisadores.

Diversos modelos também foram desenvolvidos, dentre os mais utilizados e citados em pesquisas foram os desenvolvidos por Grönroos (1984); o modelo conceitual da qualidade de serviço desenvolvido por Parasuraman, Zeithaml e Berry (1985); o modelo SERVQUAL de Parasuraman, Berry e Zeithaml (1988, 1991) e o modelo SERVPERF de Cronin e Taylor (1992, 1994). Até a presente data, o modelo SERVQUAL $(1988,1991)$ é o mais utilizado em suas dimensões, metodologia e aplicação de seus questionários.

Pinheiro (1983) aborda a reformulação conceitual da Lei de Bradford, que observa a ordem de produtividade de artigos sobre um determinado assunto, nesse caso, poderão ser observados quais os periódicos que são devotados a um assunto ou tema, contendo o número de artigos neste núcleo.

Pela bibliométria foi possível observar a quantidade de artigos publicados e em quais jornais e seu respectivo Índex $H$ estão publicados os artigos sobre qualidade de serviços. Esse indicador busca auxiliar futuras submissões, pois identifica os periódicos que possivelmente possuam linhas de interesse em trabalhos que abordem tema qualidade de serviço. O período pesquisado compreende anos de 1985 até 2011.

No Quadro 3 é possível verificar em ordem decrescente a quantidade de publicações neste período. 


\begin{tabular}{|c|l|c|}
\hline $\begin{array}{c}\text { Quantidade DE } \\
\text { Publicaçōes }\end{array}$ & \multicolumn{1}{c|}{ Periódico } & INDEX H \\
\hline 95 & $\begin{array}{l}\text { The Service Industries } \\
\text { Journal }\end{array}$ & $\mathrm{H}-19$ \\
\hline 18 & $\begin{array}{l}\text { Service Marketing Quarterly } \\
11\end{array}$ & $\mathrm{H}-05$ \\
\hline 05 & MIS Quarterly & $\mathrm{H}-82$ \\
\hline 02 & $\begin{array}{l}\text { Journal of International } \\
\text { Marketing }\end{array}$ & $\mathrm{H}-30$ \\
\hline & Health Marketing Quarterly & $\mathrm{H}-13$ \\
\hline
\end{tabular}

Quadro 3: Quantidade de publicações por periódicos no período entre 1985-2011

Fonte: Elaborado pelos autores deste artigo
Urbizagastegui (2008) informa que na literatura científica as pesquisas bibliométricas lançam mão da Lei de Lotka que busca um padrão estratificado da produção científica e essa estratificação poderá muito bem se ajustar ao modelo em estudo. Uma das análises é a verificação dos autores e produtividade de artigos, que segundo a o autor denomina de contagem direta. No Quadro 4 é possível se visualizar a produtividade pela contagem direta.

Ele também apresenta, em ordem decrescente, as publicações pela quantidade de citações, sua referência e o Index $H$ da publicação. Também é foco da pesquisa verificar quais foram as publicações mais citadas.

\begin{tabular}{|c|c|c|c|}
\hline \multicolumn{2}{|r|}{ QTD. } & REFERÊNCIA DOS ARTIGOS MAIS CITADOS & INDEX $\mathbf{H}$ \\
\hline 1 & 75 & $\begin{array}{l}\text { PARASURAMAN, A; ZEITHAML; V.A; BERRY, L.L. SERVQUAL: a multiple-item scale for measuring } \\
\text { consumer perceptions of service quality. Journal of Retailing, v. } 64, \text { n. } 1, \text { p. } 12-37,1988 \text {. }\end{array}$ & 53 \\
\hline 2 & 71 & $\begin{array}{l}\text { PARASURAMAN, A; ZEITHAML; V. A; BERRY, L. L. A conceptual model of service quality and its } \\
\text { implications for further research. Journal of Marketing, v. 49, p. 41-50, } 1985 .\end{array}$ & 99 \\
\hline 3 & 49 & $\begin{array}{l}\text { CRONIN, J.; TAYLOR, S. Measuring service quality: a reexamination and extension. Journal of } \\
\text { Marketing, v. 56, n. 3, p. 55-68, } 1992 \text {. }\end{array}$ & 99 \\
\hline 4 & 35 & $\begin{array}{l}\text { CARMAN, J.M. Consumer Perceptions of Service Quality: an Assessment of the SERVQUAL } \\
\text { Dimensions, Journal of Retailing, v. 66, p. 33-55, } 1990 .\end{array}$ & 53 \\
\hline 5 & 34 & $\begin{array}{l}\text { PARASURAMAN, A.; ZEITHAML V. A; BERRY, L. L. Refinement and reassessment of the Servqual } \\
\text { Scale, Journal of Retailing, v. } 67 \text {, n. 4, p. } 420-50,1991 .\end{array}$ & 53 \\
\hline 6 & 26 & $\begin{array}{l}\text { ZEITHAML, V., PARSURAMAN, A. BERRY, L. L. Delivering Service Quality: Balancing Customer } \\
\text { Perceptions and expectations, New York: The Free Press, } 1991 .\end{array}$ & N.S.A. \\
\hline 7 & 25 & $\begin{array}{l}\text { GRONROOS, C. Strategic management and marketing in the service sector. Helsingfors: } \\
\text { Swedish School of Economics and Business Administration. } 1982 .\end{array}$ & N.S.A. \\
\hline 8 & 25 & $\begin{array}{l}\text { BITNER, M. J. Evaluating Service Encounters: the Effects of Physical Surroundings and Employee } \\
\text { Responses, Journal of Marketing, v. 54, p. 69-82, } 1990 .\end{array}$ & 99 \\
\hline 9 & 23 & $\begin{array}{l}\text { PARASURAMAN, A.; ZEITHAML V. A; BERRY, L. L. Reassessment of expectations as a comparison } \\
\text { standard in measuring service quality: Implications for future research. Journal of Marketing, v. 58, p. } \\
\text { 111-124, } 1994 .\end{array}$ & 99 \\
\hline 10 & 23 & $\begin{array}{l}\text { TEAS, R.K. Expectations, performance evaluation, and customers perceptions of quality. Journal of } \\
\text { Marketing, 57(4), p.18-34, } 1993 .\end{array}$ & 99 \\
\hline 11 & 22 & $\begin{array}{l}\text { CRONIN, J.J; TAYLOR, S.A. SERVPERF versus SERVQUAL: Reconciling Performance Based and } \\
\text { Perception-Minus-Expectations Measurement of Service Quality', Journal of Marketing, v.58, n.1, p } \\
\text { 125-131, 1994. }\end{array}$ & 99 \\
\hline 12 & 21 & $\begin{array}{l}\text { ZEITHAML, V.A, BERRY, L.L; PARASURAMAN, A. The behavioural consequences of service quality. } \\
\text { Journal of Marketing, 60(4), 31-46. } 1996 .\end{array}$ & 99 \\
\hline 13 & 21 & $\begin{array}{l}\text { GRONROOS, C. A Service Quality Model and its Marketing Implications': European Journal of } \\
\text { Marketing, v.18, n. 4, p. 36-44, } 1984 .\end{array}$ & 19 \\
\hline 14 & 18 & $\begin{array}{l}\text { BITNER, M. J. The service encounter: diagnosing favourable and unfavourable incidents, Journal of } \\
\text { Marketing, 54(1), p.71-84. } 1990 .\end{array}$ & 99 \\
\hline 15 & 17 & $\begin{array}{l}\text { BERRY. LE; PARASURAMAN, A. Marketing Services: Competing Through Quality, Free Press, } \\
\text { New York, } 1991 .\end{array}$ & N.S.A. \\
\hline
\end{tabular}




\begin{tabular}{|c|c|c|c|}
\hline \multicolumn{2}{|r|}{ QTD. } & REFERÊNCIA DOS ARTIGOS MAIS CITADOS & INDEX $\mathbf{H}$ \\
\hline 16 & 15 & $\begin{array}{l}\text { PARASURAMAN, A., ZEITHAML, V.A.; BERRY, L.L. Alternative Scales for Measuring Service Quality: A } \\
\text { Comparative Assessment Based on Psychometric and Diagnostic Criteria, Journal of Retailing, n.70, } \\
\text { p. 201-230, } 1994 .\end{array}$ & 53 \\
\hline 17 & 15 & $\begin{array}{l}\text { LOVELOCK, C.H. Classifying Services to Gain Strategic Marketing Insights. Journal of Marketing, } \\
\text { 47, p. 9-20, } 1983 .\end{array}$ & 99 \\
\hline 18 & 15 & $\begin{array}{l}\text { ZEITHAML, V. A, BERRY, L. L; PARASURAMAN, A. The nature and determinants of customer } \\
\text { expectations of service. Journal of the Academy of Marketing Science, n. 21, p. 1-12. } 1993 .\end{array}$ & 68 \\
\hline 19 & 15 & $\begin{array}{l}\text { ZEITHAML, V. A, BERRY, L. L; PARASURAMAN, A. Communication and control processes in the } \\
\text { delivery of service quality. Journal of Marketing, v.52, p.35-48. } 1988 .\end{array}$ & 99 \\
\hline 20 & 14 & $\begin{array}{l}\text { GRONROOS, C. Service Management and Marketing - Managing the Moments of Truth in } \\
\text { Service Competition, Lanham. MD: Lexington Books. } 1990 .\end{array}$ & N.S.A. \\
\hline
\end{tabular}

N.S.A.: não se aplica

Quadro 4: Artigos que citam mais sobre qualidade de serviços

Fonte: Elaborado pelos autores deste artigo

Visando explorar não somente as obras mais citadas e as publicações em periódicos de alto impacto, com a pesquisa bibliométrica foi possível observar a formação de redes de autores. Através da rede se verifica que a autora Leyland F. Pitt foi a autora que mais apresentou relações, perfazendo dez laços de saída. O coautor mais chamado para a realização das publicações foi C. Bruce Kavan, totalizando seis laços de entrada. Na Figura 1 é apresentada a formação das diversas redes de autores no mesmo período estudado.

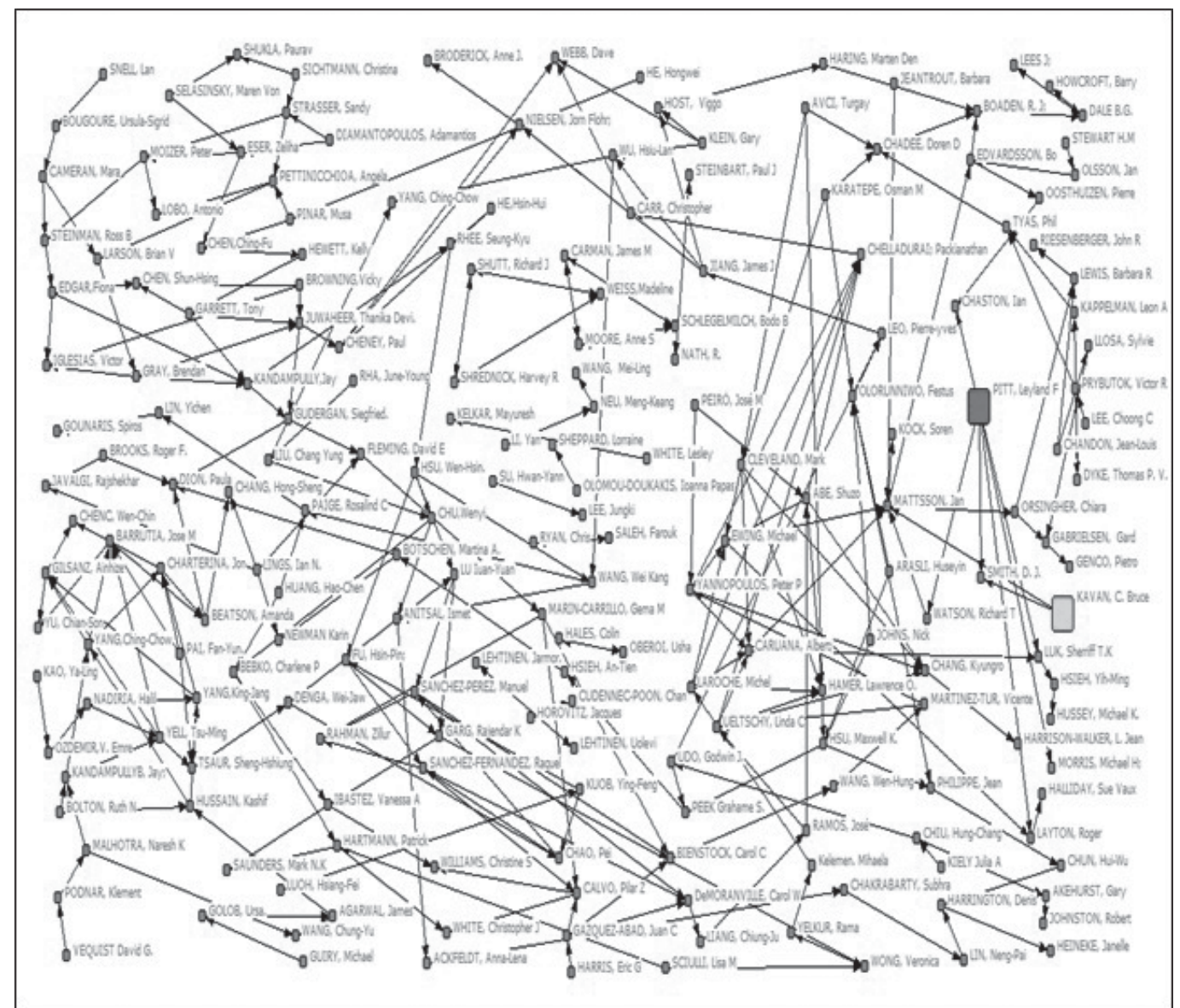

Figura 1: Rede de autores

Fonte: Elaborada pelos autores deste artigo 
Além da rede de autores, outra rede que se pode constatar foi a de instituições de ensino em diversos países. A University of Wales, no Reino Unido, é a instituição com os maiores laços de saídas, totalizando em sete ao todo, sendo a University of Georgia, nos Estados Unidos da América, a instituição com maiores laços de entradas, somando também sete.

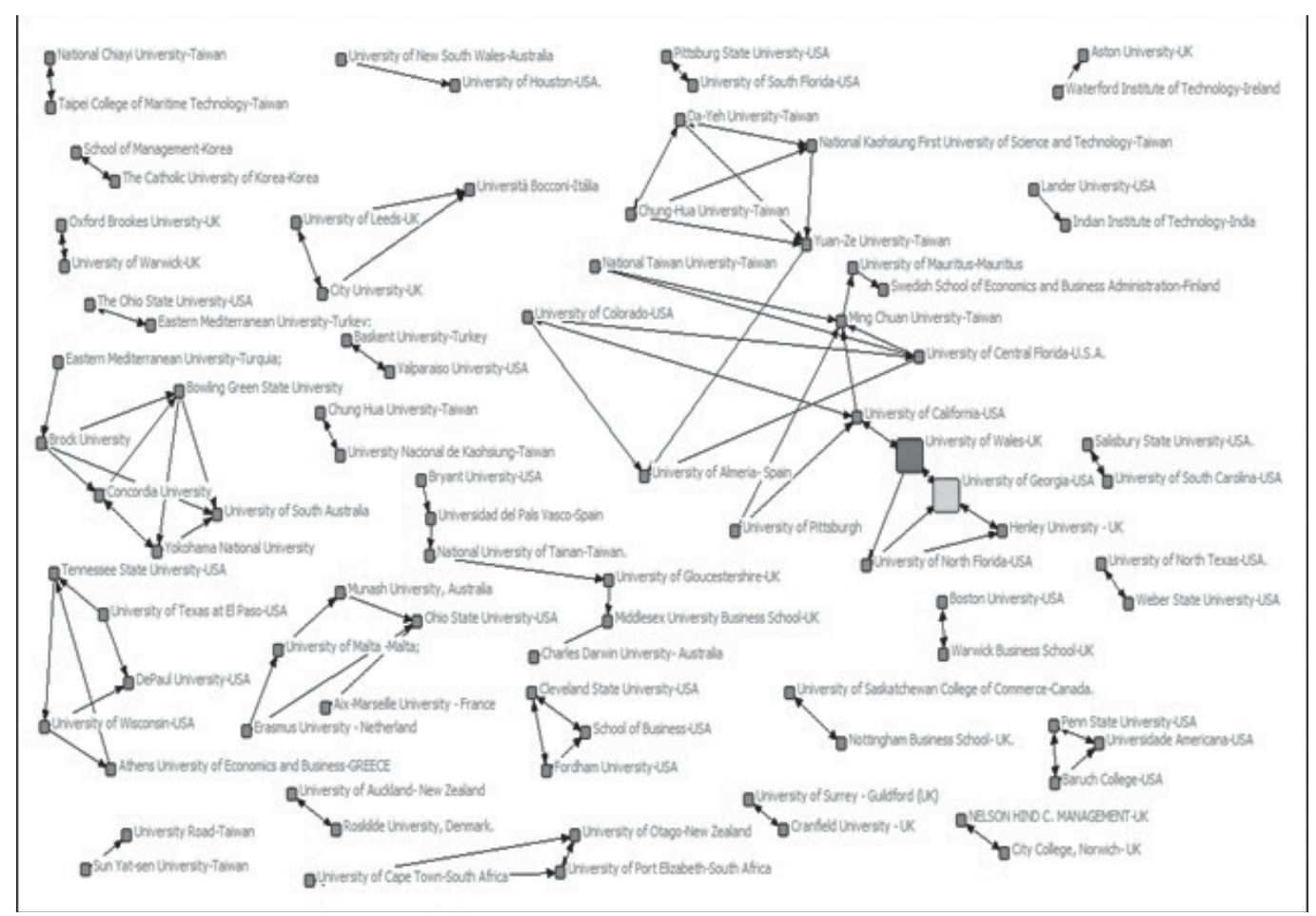

Figura 2: Rede de Instituições de Ensino Superior

Fonte: Elaborada pelos autores deste artigo

Destacam-se as palavras utilizadas para pesquisas futuras nas bases de dados internacionais, que são: service quality, marketing strategy, marketing service e service encounter.
Com o intuito de identificar quais as relações nos estudos com a qualidade de serviço e contribuir, dessa forma, com futuras pesquisas o Quadro 5 apresenta estas relações.

\begin{tabular}{|c|c|c|}
\hline Ano & Estudo & ESTUDO RELAÇÃo REALIZADA COM QUALIDADE DE SERVIÇO \\
\hline 1985 & Nightingale & Satisfação e desempenho. \\
\hline \multirow{2}{*}{1986} & Willborn & Garantias da qualidade-auditoria e desempenho. \\
\hline & Oakland & Design de serviços, custos e conformidades. \\
\hline 1987 & Collier & Níveis da Qualidade, consumidor, necessidades e desempenho. \\
\hline \multirow{3}{*}{1988} & Lees e Dale & Círculo da qualidade total, diferenciação de serviços e imagem. \\
\hline & Edvardsson & Incidentes críticos, Interação, Diferenciação e desempenho financeiro. \\
\hline & Rhyne & Satisfação, utilização, competitividade, eficiência, produtividade, e desempenho. \\
\hline \multirow{2}{*}{1989} & Callan & Dimensões do SERVQUAL (1985). \\
\hline & Armistead & Quadro de tempo, franqueza, flexibilidade, estilo, direção e Segurança. \\
\hline \multirow{2}{*}{$\begin{array}{l}1990 \\
1990\end{array}$} & Oberoi e Hales & Dimensões do SERVQUAL (1985). \\
\hline & Horovitz e Cudennec-Poon & Atendimento, Linha de frente, Comunicação e Liderança. \\
\hline
\end{tabular}




\begin{tabular}{|c|c|c|}
\hline Ano & Estudo & ESTUDO RELAÇÃO REALIZADA COM QUALIDADE DE SERVIÇO \\
\hline \multirow{2}{*}{1991} & Lehtinen e Lehtinen & Diferenciação de serviços. \\
\hline & Saleh e Ryan & Dimensões do SERVQUAL (1985). \\
\hline \multirow{5}{*}{1992} & Shrednick, Shutt e Weiss & $\begin{array}{l}\text { Diferenciação, Produtividade, Liderança, planejamento, satisfação, garantia de } \\
\text { serviços, análises e informações e Desempenho. }\end{array}$ \\
\hline & Steinbart e Nath & Característica, políticas da empresa, percepção e desempenho. \\
\hline & Laws & Orientação ao mercado. \\
\hline & Schlegelmilch, Carman e Moore & $\begin{array}{l}\text { Informações, profissionalismo, comunicação, competência técnica, } \\
\text { Problemas emocionais e inimizade percebida. }\end{array}$ \\
\hline & Howcroft & Satisfação, Pacote e característica e questões de negócios. \\
\hline \multirow{2}{*}{1993} & Boaden e Dale & Diferenciação, organização, cultura, sistemas e medição e feedback. \\
\hline & Edvardsson e Mattsson & Diferenciação, Atores, Speaker, perspectiva e objetivo. \\
\hline \multirow{4}{*}{1994} & Mattsson & Diferenciação, Segmentação, Atração, Relacionamento e Satisfação. \\
\hline & Pitt e Jeantrout & Expectativa, Habilidades, Orientação para o mercado e Desempenho. \\
\hline & Smith & Processos e Desempenho. \\
\hline & Callan & Ferramentas da qualidade Total. \\
\hline \multirow{3}{*}{1995} & Pitt, Watson e Kavan & Satisfação do consumidor. \\
\hline & Ross & Diferenciação, conflito, estresse. \\
\hline & Chaston & Estilo, operacional, Sistema de informação, estrutura e habilidades. \\
\hline \multirow{7}{*}{1996} & Edvardsson e Olsson & Diferenciação, Funcionários, Processo, Pré-requisitos e Desempenho. \\
\hline & Pitt, Morris e Oosthuizen & Expectativas e Segmentação. \\
\hline & Witt e Stewart & Satisfação e desempenho. \\
\hline & Dickens & Diferenciação, características dos serviços. \\
\hline & Holmlund, e Soren & Marketing de relacionamento, satisfação. \\
\hline & Chadee e Mattsson & Experiências, satisfação. \\
\hline & Johns e Tyas & Satisfação e dimensões da qualidade. \\
\hline \multirow{6}{*}{1997} & Dyke, Kappelman e Prybutok & Metodologia - SERVQUAL \\
\hline & Kettinger e Lee & SERVQUAL X SERVPERF \\
\hline & Pitt, Watson e Kavan & Metodologia - SERVQUAL \\
\hline & Mels, Boshoff e Nel & Percepção e Dimensões. \\
\hline & Johns e Tyas & Percepção, incidentes críticos e desempenho. \\
\hline & Genco & Economia, competição e desempenho. \\
\hline \multirow{7}{*}{1998} & Riesenberger & Diferenciação, Capital intelectual. \\
\hline & Watson, Pitt e Kavan & Diferenciação e níveis organizacionais (estratégico, tático e operacional) \\
\hline & Lewis e Gabrielsen & Diferenciação, Estratégias, Marketing interno e Empowerment. \\
\hline & Llosa, Chandon e Orsingher & Metodologia SERVQUAL \\
\hline & Foster, e Newman & Diferenciação e Metodologia SERVQUAL. \\
\hline & Johnston e Heineke & Percepção e desempenho. \\
\hline & Dion, Javalgi e Dilorenzo-Aiss & Nível Serviço, promessas, intensificadores, satisfação e desconfirmação. \\
\hline
\end{tabular}




\begin{tabular}{|c|c|c|}
\hline Ano & Estudo & ESTUDO RELAÇÃO REALIZADA COM QUALIDADE DE SERVIÇO \\
\hline \multirow{5}{*}{1999} & Gould-Willims & Diferenciação, lealdade e desempenho. \\
\hline & Haring e Mattsson & Lingüística e psicologia social. \\
\hline & Broderick & Diferenciação, teoria dos papeis e encontro de serviços. \\
\hline & Brooks, Lings e Botschen & Diferenciação, Modelo SERVQUAL, Mkt interno. \\
\hline & Hussey & Modelos SERQUAL e SERVPERF. \\
\hline \multirow{5}{*}{2000} & Harrington e Akehurst & Estratégia, comportamento do gestor. \\
\hline & Webb & Satisfação dos clientes, desempenho. \\
\hline & Nielsen e Host & Diferenciação e Zona de tolerância. \\
\hline & Smith & Diferenciação, satisfação. \\
\hline & Halliday & Zona de tolerância, Confiança, satisfação, expectativas. \\
\hline \multirow{2}{*}{2001} & Hsieh e Hsieh & Padronização dos processos. \\
\hline & Holmlund & Dimensões e percepção. \\
\hline \multirow{7}{*}{2002} & Chiu e Wu & Cognição, afeição e comportamento. \\
\hline & Jiang, Klein e Carr & Modelo SERVQUAL e Satisfação. \\
\hline & Harrison-Walker & Dimensões do SERVQUAL. \\
\hline & Luk, e Layton & Zona de tolerância. \\
\hline & Kiely e Peek & Percepção e Cultura. \\
\hline & Léo e Philippe & Diferenciação, satisfação, \\
\hline & Illeris & Diferenciação, competência (educação, habilidades e atitudes). \\
\hline \multirow{5}{*}{2003} & Chang e Chelladurai & Clima, gestão, Hab. interpessoais, ambiente, e interação com clientes. \\
\hline & Caruana, Pitt e Ewing & Orientação para mercado e Desempenho. \\
\hline & Hamer & Métodos, vantagens e desvantagens. \\
\hline & Olorunniwo, Hsu e Udo & Dimensões da qualidade. \\
\hline & Sheppard & Diferenciação, interação pessoal. \\
\hline \multirow{5}{*}{2004} & Laroche et al. & Satisfação. \\
\hline & Johns, Avci e Karatepe & Modelos SERVQUAL e SERVPERF. \\
\hline & Chiu, e Lin & Psicológica, Segurança, Afeição, Auto-atualização, Conhec. e Estética. \\
\hline & $\begin{array}{l}\text { Kelemen e Papasolomou } \\
\text { Doukakis }\end{array}$ & Cultura, Cultura organizacional, imaginário do consumidor. \\
\hline & Karatepe et al. & Diferenciação, padronização serviço e satisfação com o trabalho. \\
\hline \multirow{4}{*}{2005} & Kettinger e Lee & Zona de tolerância, expectativas. (percebida, desejado e expectativas). \\
\hline & Peiró et al. & Lealdade, qualidade funcional e qualidade relacional. \\
\hline & Gounaris & Dimensões do modelo SERVQUAL. \\
\hline & Lee & Modelo SERVQUAL. \\
\hline
\end{tabular}




\begin{tabular}{|c|c|c|}
\hline Ano & Estudo & ESTUDO RELAÇÃO REALIZADA COM QUALIDADE DE SERVIÇO \\
\hline \multirow{11}{*}{2006} & Yelkur e Chakrabarty & Expectativa, segmentação e dimensões do SERVQUAL. \\
\hline & White & Humor e Lealdade. \\
\hline & Williams e Saunders & Estudou a evolução da qualidade de serviços. \\
\hline & Liang e Wang & Satisfação, Lealdade, MKT de Relacionamento e percepção. \\
\hline & Su e Lin & Gestão do conhecimento, vantagem competitiva. \\
\hline & Ackfeldt e Wong & Diferenciação, Satisfação no trabalho, Comprometimento. \\
\hline & Ibáñez, Hartmann e Calvo & Diferenciação, Percepção, Satisfação, Fidelização/Lealdade. \\
\hline & Bienstock e Demoranville & Diferenciação, comportamentos de cidadania organizacional. \\
\hline & Rahman & SERVQUAL, Vantagem competitiva, desempenho. \\
\hline & Anitsal e Paige & Diferenciação, auto-atendimento, self-checkout, e quiosques eletrônicos. \\
\hline & Bebko, Sciulli e Garg & Expectativa do IDEAL e a percepção do ACEITÁVEL. \\
\hline \multirow{5}{*}{2007} & Sanchez-Perez et al. & Natureza do serviço, Mensuração da qualidade, \\
\hline & Huang, Chu e Wang & BSC e Desempenho. \\
\hline & Yang & Geração e conceito de serviço, análise de negócios e decisão estratégica. \\
\hline & Chao, Fu e Lu & Níveis de Lealdade, Orientação de mercado, \\
\hline & Harris e Fleming & 5 fatores de personalidade e satisfação. \\
\hline \multirow{6}{*}{2008} & Chang & Valor para o consumidor (maior estratégia de diferenciação). \\
\hline & Wu, Liu e Hsu & Valor percebido, Satisfação e Intenções comportamentais. \\
\hline & Chao & Diferenciação - Lealdade, relacionamento e atributos. \\
\hline & Deng, Kuo e Chenc & Análise Importância-desempenho, benchmarking e satisfação \\
\hline & $\mathrm{Yu}$ & Diferenciação, mensuração da qualidade, importância e satisfação. \\
\hline & Beatson, Lings e Gudergan & Satisfação, Confiança e Compromisso. \\
\hline \multirow{15}{*}{2009} & Klein, Jiang e Cheney & Análise de medidas indiretas \\
\hline & Rhee e Rha & Satisfação, Acidentes críticos, processo, design e relacionamento. \\
\hline & Luoh e Tsaur & Diferenciação, cenários de qualidade e as aparições de servidor. \\
\hline & Barrutia, Charterina e Gilsanz & Comercialização, tecnologia, marketing interno e desempenho. \\
\hline & Hu, Kandampully e Juwaheer & Satisfação; Valor percebido; Imagem e lealdade. \\
\hline & Chen & Diferenciação - Importância, satisfação e desempenho. \\
\hline & Iglesias & Falha no serviço, atribuição, satisfação e processamento de informação. \\
\hline & Yang et al. & Satisfação do cliente, índice de melhoria e pesquisa com o cliente. \\
\hline & Davies & Diferenciação, processos, relações interpessoais e tolerância. \\
\hline & Browning et al. & Diferenciação, desempenho, dimensão em equipe. \\
\hline & $\begin{array}{l}\text { Nadiri, Kandampullyb e } \\
\text { Hussain }\end{array}$ & Zona de tolerância, natureza dos serviços e expectativa. \\
\hline & Wang & Valor percebido, Imagem corporativa e as barreiras para mudança \\
\hline & Sharif & Interserviços, qualidade e cadeia de lucro serviço. \\
\hline & Lobo & Fidelização, adequação do serviço, Satisfação e Zonas de tolerância. \\
\hline & Larson e Steinman & Intenções de compra, satisfação e marketing esportivo. \\
\hline
\end{tabular}




\begin{tabular}{|c|c|c|}
\hline Ano & Estudo & ESTUDO RELAÇÃO REALIZADA COM QUALIDADE DE SERVIÇO \\
\hline \multirow{11}{*}{2010} & Ozdemir, e Hewett & Relacionamento, Intenções, Coletivismo, Compromisso e Confiança. \\
\hline & Agarwal, Malhotra e Bolton & Diferenciação, atitudes, segmentação \\
\hline & Wang & Práticas de gestão de $\mathrm{RH}$ e papel da cidadania. \\
\hline & Cameran, Moizer e Pettinicchio & Imagem corporativa e Satisfação. \\
\hline & Chen e Kao & Processo, resultado, satisfação e intenções comportamentais. \\
\hline & Podnar e Golob & Marketing interno; flexibilidade, trabalho, orientação aos serviços. \\
\hline & Kelkar & Modelo SERVQUAL x Modelo SERVDIV. \\
\hline & Bougoure e Neu & Percepção, Satisfação, Intenções de recompra. \\
\hline & Kassim e Zain & Percepção e Expectativa. \\
\hline & Pinar, Eser e Strasser & Benchmarking, cliente misterioso. \\
\hline & Shukla & Lealdade, satisfação, sacrifício percebido e Valor percebido. \\
\hline \multirow{4}{*}{2011} & Guiry e Vequist & Cuidados de saúde, Turismo de saúde, Turismo médico e SERVQUAL. \\
\hline & Snell e White & Sociocognitiva, psicossocio, inteligência emocional e competência. \\
\hline & He e Li & Fidelidade da marca, Satisfação, identificação e associação da marca. \\
\hline & $\begin{array}{l}\text { Sichtmann, Selasinsky e } \\
\text { Diamantopoulos }\end{array}$ & Diferenciação, desempenho do funcionário, processos e desempenho organizacional. \\
\hline
\end{tabular}

Quadro 5: Variáveis utilizadas para mensuração da qualidade de serviço

Fonte: Elaborado pelos autores deste artigo

Na Figura 3 pode-se verificar a distribuição da quantidade de publicação por ano, considerando os anos de 1985 até 2011. O destaque fica para o ano de 2009 com 15 publicações no ano, e também para os anos de 2006 e 2010 com 11 publicações em jornais de alto impacto mundial.
Assim, com base na revisão bibliográfica e nos procedimentos metodológicos anteriormente apresentados, além da análise bibliométrica sobre a qualidade de serviços, serão apresentadas nas considerações finais as sugestões para trabalhos futuros.

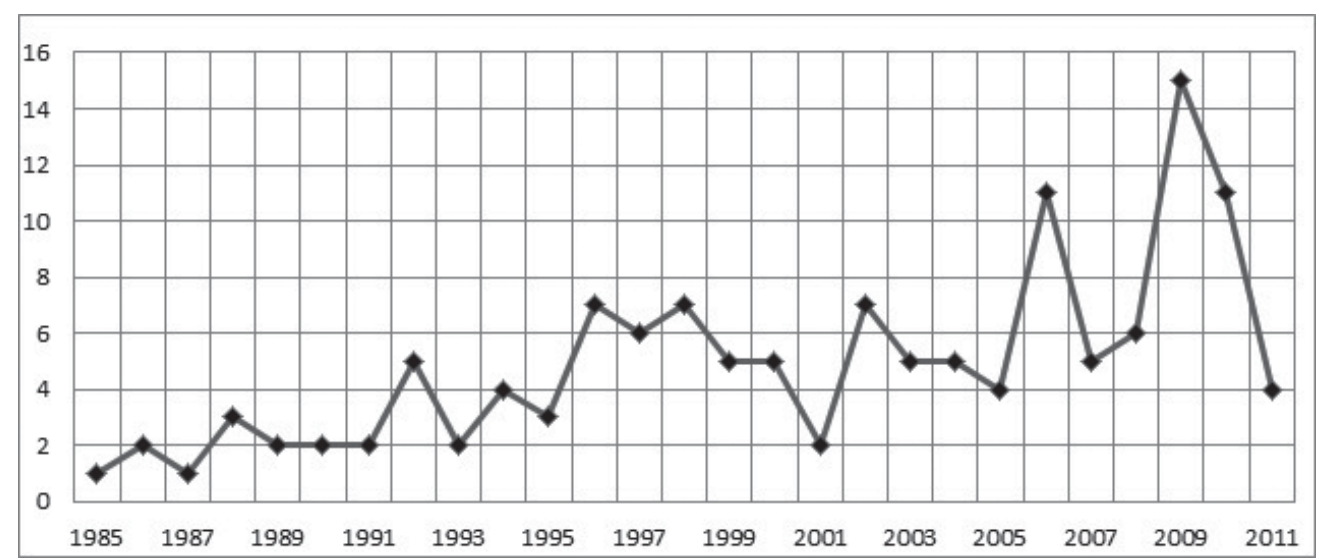

Figura 3: Quantidade de artigos publicados de 1985 até 2011

Fonte: Elaborada pelos autores deste artigo 


\section{Considerações Finais, Limitações e Recomendações de Estudo}

No decorrer deste artigo, diversas análises foram realizadas no intuito de explorar o estado da arte sobre qualidade de serviços, tendo como método a bibliometria. A partir da análise de 132 publicações ao longo do período de 1985 a 2011, pode-se observar o destaque de algumas contribuições desta pesquisa.

Contribuições verificadas na bibiométrica: i) o primeiro autor mais citado é Parasuraman com 255 referências; ii) A metodologia mais utilizada é o SERVQUAL (1988, 1991); iii) a publicação mais referenciada é a obra A multiple-item scale for measuring consumer perceptions of service quality, publicado no Journal of Retailing, em 1988, com 75 citações; iv) dentre os periódicos, observou-se que o The Service Industries Journal obteve destaque no número de artigos publicados com 95 publicações. Esse tipo de informação é oportuna aos pesquisadores que se dedicam a publicar sobre o tema em jornal de alto impacto; v) o ano de 2009 foi o de maior publicações sobre qualidade de serviço com 15 publicações, demonstrando que o tema ainda é discutido pela ciência; vi) a rede de autores apresenta que Leyland Pitt é a autora com dez laços de saída, isto é, ela é a autora com maior diversidade de coautores; e o coautor mais chamado foi $\mathrm{C}$. Bruce Kavan, totalizando seis laços de entrada; vii) a rede de instituições que a University of Wales, no Reino Unido, é a instituição com maior laços de saídas, e a University of Georgia, nos Estados Unidos da América, a instituição com maior laços de entradas, ambas com sete relações; viii) são informados os termos utilizados buscando facilitar futuros pesquisadores, sendo eles: service quality, marketing strategy, marketing service e service encounter; ix) os estudos e as relações apresentadas dos 132 artigos pesquisados; $\mathrm{x}$ ) os critérios de Hamer (2003), sobre o objetivo e os critérios a serem utilizados, dependendo do objetivo da pesquisa, a metodologia é mais apropriada ao referido estudo; xi) o paradigma da desconfirmação continua sendo o mais popular nas pesquisas sobre qualidade de serviços em diversos segmentos como educação, bancos, saúde dentre outros; no presente estudo é apresentado 160 obras a serem pesquisadas.
A principal limitação deste estudo se dá pelo fato de a base de dados ser limitada a alguns periódicos e aos filtros metodológicos dos autores, que pode acarretar em deixar às margens da coleta algum trabalho relevante.

Para trabalhos futuros recomenda-se a ampliação deste estudo identificando outras variáveis, que neste não foram possíveis de serem estudadas, como por exemplo, a aplicação de alguma técnica de análise multivariada, tal como análise de correspondência múltipla que poderá identificar os estudos que possuem correlação. Outra recomendação reside na realização de um estudo similar nas bases de dados nacionais.

Por fim, procurou-se abordar alguns aspectos que, certamente, merecem atenção futura por parte dos pesquisadores e dos gestores das organizações. Dessa forma, o esforço realizado para apresentar alguns elementos importantes dos estudos sobre qualidade de serviços será mais uma contribuição na direção de discussões mais profundas acerca do assunto.

\section{REFERÊNCIAS}

ACKFELDT, A. L.; WONG, V. The Antecedents of Prosocial Service Behaviours: An Empirical Investigation. The Service Industries Journal, United Kingdom, v. 26, n. 7, p. 727-745, 2006.

ANITSAL, I.; PAIGE, R. C. An Exploratory Study on Consumer Perceptions of Service Quality in Technology Based Self-Service. Service Marketing Quarterly, United Kingdom, v. 27, n. 3, p. 53-67, 2006.

ARAUJO, W. T. et al. Meta-análise das dissertações do curso de mestrado ciência da informação UFPB: 19901999. Revista Informação e Sociedade: estudos. João Pessoa, v. 10, n. 1, 2000.

ARMISTEAD, C. G. Customer Service and Operations Management in Service Businesses. The Service

Industries Journal, United Kingdom, v. 9, n. 2, p. $247-$ 260, 1989.

BARRUTIA, J. M; CHARTERINA, J.; GILSANZ, A.

E-service quality: an internal, multichannel and pure service perspective. The Service Industries Journal, United Kingdom, v. 29, n. 12, p. 1.707-1.721, 2009. 
BEATSON, A.; LINGS, I.; GUDERGAN, S. Employee behavior and relationship quality: impacto $n$ customers. The Service Industries Journal, United Kingdom, v. 28, n. 2, p. 211-223, 2008.

BEBKO, C. P.; SCIULLI, L. M.; GARG, R. K. Consumers' Level of Expectation for Services and the Role of Implicit Service Promises. Service Marketing Quarterly, United Kingdom, v. 28, n. 2, p. 1-23, 2006.

BERRY, L. E.; PARASURAMAN, A. Marketing services: competing through quality. New York: Free Press, 1991.

BIENSTOCK, C. C.; DeMORANVILLE, C. W. Using Manager Reports of Employee Behavior to Investigate the Relationship Between Organizational Citizenship Behaviors and Customers' Perceptions of Service Quality. Service Marketing Quarterly, United Kingdom, v. 26, n. 1, p. 103-118, 2006.

BITNER, M. J. Evaluating Service Encounters: the Effects of Physical Surroundings and Employee Responses, Journal of Marketing, Chicago: AMA, v. 54, p. 69-82, 1990.

BITNER, M. J. The service encounter: diagnosing favourable and unfavourable incidents, Journal of Marketing, Chicago: AMA, v. 54, p. 71-84. 1990.

BOADEN, R. J; DALE, B. G. Managing Quality Improvement in Financial Services: A Framework and Case Study. The Service Industries Journal, United Kingdom, v. 13, n. 1, p. 17-39, 1993.

BOUGOURE, U. S.; NEU, M. K. Service Quality in the Malaysian Fast Food Industry: An Examination Using DINESERV. Service Marketing Quarterly, United Kingdom, v. 31, n. 2, p. 194-212, 2010.

BOULDING, W. et al. A dynamic process model of service quality: from expectations to behavioral intentions.

Journal of marketing research, Chicago: AMA, v. 30, p. 7-27, 1993.

BRODERICK, A. J. Role Theory and the Management of Service encounters. The Service Industries Journal, United Kingdom, v. 19, n. 2, p. 117-131, 1999.

BROOKS, R. F; LINGS, I. N.; BOTSCHEN, M. A. Internal Marketing and Customer Driven Wavefronts. The Service Industries Journal, United Kingdom, v. 19, n. 4, p. 117-131, 1999.
BROWNING, V. et al. Realising competitive advantage through HRM in New Zealand service industries. The Service Industries Journal, United Kingdom, v. 29, n. 6, p. 741-760, 2009.

CALLAN, R. J. Small Country Hotels and Hotel Award Schemes as a Measurement of Service Quality. The Service Industries Journal, v. 9, n. 2, p. 223-246, 1989.

CALLAN, R. J. Quality Assurance Certification for Hospitality Marketing, Sales and Customer Services. The Service Industries Journal, United Kingdom, v. 14, n. 4, p. 482-498, 1994.

CAMERAN, M.; MOIZER, P.; PETTINICCHIOA, A. Customer satisfaction, corporate image, and service quality in professional services. The Service Industries Journal, United Kingdom, v. 30, n. 3, p. 421-435, 2010.

CARMAN, J. M. Consumer Perceptions of Service Quality: An Assessment of the SERVQUAL Dimensions, Journal of Retailing, Cambridge, MA, USA, v. 66, p. 33-55, 1990.

CARUANA, A.; PITT, L.; EWING, M. The Market Orientation - Performance Link: The Role of Service Reliability. The Service Industries Journal, United Kingdom, v. 23, n. 4, p. 25-41, 2003.

CHADEE, D. D.; MATTSSON, J. An Empirical Assessment of Customer Satisfaction in Tourism. The Service Industries Journal, United Kingdom, v. 16, n. 3, p. 305-320, 1996.

CHANG, K.; CHELLADURAI; P. System-Based Quality Dimensions in Fitness Services: Development of the Scale of Quality. The Service Industries Journal, United Kingdom, v. 23, n. 5, p. 65-83, 2003.

CHANG, H. S. Increasing hotel customer value through service quality cues in Taiwan. The Service Industries Journal, United Kingdom, v. 28, n. 1, p. 73-84, 2008.

$\mathrm{CHAO}, \mathrm{P}$. Exploring the nature of the relationships between service quality and customer loyalty: an attributelevel analysis. The Service Industries Journal, United Kingdom, v. 28, n. 1, p. 95-116, 2008. 
CHAO, P.; FU; H. P.; LU, L. Y. Strengthening the Quality - Loyalty Linkage: The Role of Customer Orientation and Interpersonal Relationship. The Service Industries Journal, United Kingdom, v. 27, n. 4, p. 471-494, 2007.

CHASTON, I. A Typology for Evaluating Branch-Level Perceptions of Internal Customer Management Processes within the UK Clearing Banks. The Service Industries Journal, United Kingdom, v. 15, n. 3, p. 332-349, 1995.

CHEN, C. F; KAO, Y. L. Relationships between process quality, outcome quality, satisfaction, and behavioural intentions for online travel agencies - evidence from Taiwan. The Service Industries Journal, United Kingdom, v. 30, n. 12, p. 2.081-2.092, 2010.

CHEN, S. H. Establishment of a performance-evaluation model for service quality in the banking industry. The

Service Industries Journal, United Kingdom, v. 29, n. 2, p. 235-247, 2009.

CHIU, H. C.; LIN, N. P. A Service Quality Measurement Derived from the Theory of Needs. The Service

Industries Journal, United Kingdom, v. 24, n. 1, p. 187204, 2004.

CHIU, H. C.; WU, H. C. Exploring the Cognitive and Affective Rolesof Service Quality Attitude across Gender. The Service Industries Journal, United Kingdom, v. 22, n. 3, p. 63-76, 2002.

COLLIER, D. A. The Customer Service and Quality Challenge. The Service Industries Journal, United Kingdom, v. 7, n. 1, p. 77-90, 1987.

CRONIN, J.; TAYLOR, S. Measuring service quality: A reexamination and extension. Journal of Marketing, $v$. 56, n. 3, p. 55-68, 1992.

CRONIN, J. J.; TAYLOR, S. A. SERVPERF versus SERVQUAL: Reconciling Performance Based and Perception-Minus-Expectations Measurement of Service Quality', Journal of Marketing, Chicago: AMA, v. 58, n. 1, p. 1-25, 1994 .

DAVIES, M. Zone of tolerance for Banks: a diagnostic model of service quality. The Service Industries Journal, United Kingdom, v. 29, n. 1, p. 91-110, 2009.
DENG, W. J.; KUO, Y. F.; CHEN, W. C. Revised importance - performance analysis: three-factor theory and benchmarking. The Service Industries Journal, United Kingdom, v. 28, n. 1, p. 37-51, 2008.

DICKENS, P. Human Service as service Industries. The Service Industries Journal, United Kingdom, v. 16, n. 1, p. 82-91, 1996.

DION, P.; JAVALGI, R.; DILORENZO-AISS, J. The Hospitality Industry: Defining Quality for a Quality Assurance Programme - A Study of Perceptions. The Service Industries Journal, United Kingdom, v. 18, n. 4, p. 66-86, 1998.

DYKE, T. P. V.; KAPPELMAN, L. A.; PRYBUTOK, V. R. Measuring Information Systems Service Quality: Concerns on the Use of the SERVQUAL Questionnaire. MIS Quarterly, USA, v. 22, n. 1, p. 195-208, 1997.

EDVARDSSON, B. Service Quality in Customer Relationships: A study of critical incidents in mechanical engineering companies. The Service Industries Journal, United Kingdom, v. 8, n. 4, p. 427-445, 1988.

EDVARDSSON, B.; MATTSSON, J. An ExperiencedBased Measure of Service Quality. The Service Industries Journal, United Kingdom, v. 13, n. 4, p. 289-306, 1993.

EDVARDSSON, B.; OLSSON, J. Key Concepts for New Service Development. The Service Industries Journal, United Kingdom, v. 16, v. 2, p. 140-164, 1996.

FOSTER, G.; NEWMAN, K. What is Service Quality When 'Service' Equals Regulation? The Service Industries Journal, United Kingdom, v. 18, n. 4, p. 51-65, 1998.

GARWAL, J.; MALHOTRA, N. K; BOLTON, R. N. A Cross-National and Cross-Cultural Approach to Global Market Segmentation: An Application Using Consumers' Perceived Service Quality. Journal of International Marketing, United Kingdom, v. 18, n. 3, p. 18-40, 2010. GENCO, P. Services in a Changing Economic Environment. The Service Industries Journal, United Kingdom, v. 17, n. 4, p. 529-543, 1997. 
GLENN F. R. Interpersonal Stress Reactions and Service Quality Responses among Hospitality Industry Employees. The Service Industries Journal, United Kingdom, v. 15, n. 3, p. 314-331, 1995.

GOULD-WILLIMS, J. The Impact of Employee

Performance Cues on Guest Loyalty, Perceived and Service Quality. The Service Industries Journal, United Kingdom, v. 19, n. 3, p. 97-118, 1999.

GOUNARIS, S. An Alternative Measure for Assessing Perceived Quality of Software House Services. The Service Industries Journal, United Kingdom, v. 25, n. 6, p. 803-823, 2005.

GREY, C. Reinventing business schools: the contribution of critical management education. Academy of Management Learning and Education, New York, v. 3, n. 2, p. 178-186, 2004.

GRÖNROOS, C. A Service Quality Model and its Marketing Implications: European Journal of Marketing, Bradford, v. 18, n. 4, p. 36-44, 1984.

GRÖNROOS, C. Marketing services: the case of a missing product. Journal of Business and Industrial Marketing, Georgia State, USA, v. 13, n. 4/5, p. 322338, 1998.

GRÖNROOS, C. Service Management and Marketing - Managing the Moments of Truth in Service Competition, Lanham. MD: Lexington Books. 1990.

\section{GRÖNROOS, C. Strategic management and} marketing in the service sector. Helsingfors: Swedish School of Economics and Business Administration, 1982.

GUIRY, M.; VEQUIST, D. G. Traveling Abroad for Medical Care: U.S. Medical Tourists Expectations and Perceptions of Service Quality. Health Marketing Quarterly, London, UK, AMA, v. 28, n. 3, p. 253-269, 2011.

HALLIDAY, S. V. Maternity Care: Ways to Add Value. The Service Industries Journal, United Kingdom, v. 20, n. 4, p. 131-146, 2000.

HAMER, Lawrence O. A Pragmatic View of Perceived Service Quality Measurement. Services Marketing Quarterly, London, UK, v. 24, n. 3, p. 29-42, 2003.
HARING, M. D.; MATTSSON, J. A Linguistic Approach to Studying Quality of Face-to-Face Communication. The Service Industries Journal, United Kingdom, v. 19, n. 2, p. 28-48, 1999.

HARRINGTON, D.; AKEHURST, G. An Empirical Study of Service Quality Implementation. The Service Industries Journal, United Kingdom, v. 20, n. 2, p. 133-156, 2000.

HARRIS, E. G.; FLEMING, D. E. Examining EmployeeService Personality Congruence: The Role of the FiveFactor Model and Influences on Job Outcomes. Service Marketing Quarterly, London, UK, v. 28, n. 3, p. 97-115, 2007.

HARRISON-WALKER, L. J. Examination of the Factorial Structure of Service Quality: A Multi-Firm Analysis. The Service Industries Journal, United Kingdom, v. 22, n. 2, p. 59-72, 2002.

HE, Hongwei; LI, Yan. CSR and Service Brand: The Mediating Effect of Brand Identification and Moderating Effect of Service Quality. Journal of Business Ethics, Springer, Netherlands, v. 100, n. 4, p. 673-688, 2011.

HOLMLUND, M. The D\&D Model - Dimensions and Domains of Relationship Quality Perceptions. The Service Industries Journal, United Kingdom, v. 21, n. 3, p. 13-36, 2001.

HOLMLUND, M.; KOCK, S. Relationship Marketing: The Importance of Customer-Perceived Service Quality in Retail Banking. The Service Industries Journal, United Kingdom, v. 16, n. 3, p. 287-304, 1996.

HOROVITZ, J.; CUDENNEC-POON, C. Putting Service Quality into Gear. The Service Industries Journal, United Kingdom, v. 10, n. 2, p. 249-265, 1990.

HOWCROFT, B. Customer Service in Selected Branches of a UK Clearing Bank. The Service Industries

Journal, United Kingdom, v. 12, n. 1, p. 125-142, 1992.

HSIEH, Y. M.; HSIEH, A. T. Enhancement of service quality with job standartion. The Service Industries Journal, United Kingdom, v. 21, n. 3, p. 147-166, 2001. 
HU, H. H.; KANDAMPULLY, J.; JUWAHEER, T. D.

Relationships and impacts of service quality, perceived value,customer satisfaction, and image: an empirical study. The Service Industries Journal, United Kingdom, v. 29, n. 2, p. 111-125, 2009.

HUANG, H. C.; CHU, W.; WANG, W. K. Strategic Performance Measurement and Value Drivers: Evidence from International Tourist Hotels in an Emerging Economy. The Service Industries Journal, United Kingdom, v. 27, n. 8, p. 1.111-1.128, 2007.

HUSSEY, M. K. Using the Concept of Loss: An Alternative SERVQUAL Measure. The Service Industries Journal, United Kingdom, v. 19, n. 4, p. 89-101, 1999.

IBASTEZ, V. A; HARTMANN, P.; CALVO, P. Z. Antecedents of Customer Loyalty in Residential Energy Markets: Service Quality, Satisfaction, Trust and Switching Costs. The Service Industries Journal, United Kingdom, v. 26, n. 6, p. 633-650, 2006.

IGLESIAS, V. The attribution of service failures: effects on consumer satisfaction. The Service Industries Journal, United Kingdom, v. 29, n. 2, p. 127-141, 2009.

ILLERIS, Sven. Are Service Jobs as Bad as Theory Says? Some Empirical Findings from Denmark. The Service Industries Journal, United Kingdom, v. 22, n. 4, p. 1-18, 2002.

JIANG, J. J.; KLEIN, G.; CARR, C. Measuring Information System Service Quality: SERVQUAL From the Other Side. MIS Quarterly, University of Minnesota, USA, v. 26, n. 2, p. 147-166, 2002.

JOHNS, N.; AVCI, T; KARATEPE, O.M. Measuring Service Quality of Travel Agents: Evidence from Northern Cyprus. The Service Industries Journal, United Kingdom v. 24, n. 3, p. 82-100, 2004.

JOHNS, N.; TYAS, P. Customer Perceptions of Service Operations: Gestalt, Incident or Mythology? The Service Industries Journal, United Kingdom, v. 17, n. 3, p. 474 488, 1997.

JOHNS, N.; TYAS, P. Use of Service Quality Gap Theory to Differentiate between Food service Outlets. The

Service Industries Journal, United Kingdom, v. 16, n. 3, p. 321-346, 1996.
JOHNSTON, R.; HEINEKE, J. Exploring the Relationship between Perception and Performance: Priorities for Action. The Service Industries Journal, United Kingdom, v. 18, n. 1, p. 101-112, 1998.

KARATEPE, O. M; AVCI, T.; ARASLI, H. Effects of Job Standardization and Job Satisfaction on Service Quality: A Study of Frontline Employees in Northern Cyprus. Services Marketing Quarterly, London, UK, v. 25, n. 3, p. 1-17, 2004.

KASSIM, N.M.; ZAIN, M. Service Quality: Gaps in the College of Business. Service Marketing Quarterly, London, UK, v. 31, n. 2, p. 235-252, 2010.

KELEMEN, M.; PAPASOLOMOU-DOUKAKIS, I. Can Culture Be Changed? A Study of Internal Marketing. The Service Industries Journal, United Kingdom, v. 24, n. 5, p. 121-135, 2004.

KELKAR, M. SERVDIV: a Vedic Approach to Measurement of Service Quality. Service Marketing Quarterly, London, UK, v. 31, n. 4, p. 420-433, 2010.

KETTINGER, W. J.; LEE, C. C. Pragmatic perspectives on the measurement of information systems service quality. MIS Quarterly, University of Minnesota, USA, v. 21, n. 2, p. 223-240, 1997.

KETTINGER, W. J.; LEE, C. C. Zones of tolerance: alternative scales for measuring information systems service quality. MIS Quarterly, University of Minnesota, USA, v. 29, n. 4, p. 607-623, 2005.

KIELY, J. A.; PEEK, G. S. The Culture of the British Police: Views of Police Officers. The Service Industries Journal, United Kingdom, v. 22, n. 1, p. 167-183, 2002.

KLEIN, G.; JIANG, J. J.; CHENEY, P. Resolving difference score issues in information systems research. MIS

Quarterly, University of Minnesota, USA, v. 33, n. 4, p. 811-826, 2009.

LAROCHE, M. et al. Service Quality Perceptions and Customer Satisfaction: Evaluating the Role of Culture. Journal of International Marketing, United States of America: AMA, v. 12, n. 3, p. 58-85, 2004. 
LARSON, B. V.; STEINMAN, R. B. Driving NFL Fan

Satisfaction and Return Intentions With Concession Service Quality. Service Marketing Quarterly. London, UK, v. 30, n. 4, p. 39-53, 2009.

LAWS, E. Tourist Marketing: Service and Quality Management Perspectives. The Service Industries Journal, United Kingdom, v. 1, n. 1, p. 295-298, 1992.

LEE, J. Measuring Service Quality in a Medical Setting in a Developing Country: The Applicability of SERVQUAL.

Service Marketing Quarterly, London, UK, v. 27, n. 2, p. 1-14, 2005.

LEES, J.; DALE, B. G. Quality Circles in Service Industries: a study of their use. The Service Industries Journal, United Kingdom, v. 8, n. 1, p. 143-154, 1988.

LEHTINEN, U.; LEHTINEN, J. R. Two Approaches to Service Quality Dimensions. The Service Industries Journal, United Kingdom, v. 11, n. 3, p. 287-303, 1991.

LEO, P. Y.; PHILIPPE, J. Retail Centres: location and consumer's satisfaction. The Service Industries Journal, United Kingdom, v. 22, n. 1, p. 122-146, 2002.

LEWIS, B. R.; GABRIELSEN, G. O. S. Intra-organisational Aspects of Service Quality Management: the employees' perspective. The Service Industries Journal, United Kingdom, v. 18, n. 2, p. 64-89, 1998.

LIANG, C. J.; WANG, W. H. The Behavioural Sequence of the Financial Services Industry in Taiwan: Service Quality, Relationship Quality and Behavioural Loyalty. The Service Industries Journal, United Kingdom, v. 26, n. 2, p. 119-145, 2006.

LLOSA, S.; CHANDON, J. L.; ORSINGHER, C. An Empirical Study of SERVQUAL's Dimensionality. The Service Industries Journal, United Kingdom, v. 18, n. 2, p. 16-44, 1998.

LOBO, A. Zone of Tolerance as an Effective Management Tool to Assess Service Quality in Singapore's Stockbroking Industry. Service Marketing Quarterly, London, UK, v. 30, n. 1, p. 39-53, 2009.

LOVELOCK, C. H. Classifying Services to Gain Strategic Marketing Insights. Journal of Marketing, Chicago: AMA, v. 47, p. 9-20, 1983.
LUK, S. T. K.; LAYTON, R. Perception Gaps in Customer Expectations: Managers Versus Service Providers and Customers. The Service Industries Journal, United Kingdom, v. 22, n. 2, p. 109-128, 2002.

LUOH, H. F; TSAUR, S. H. Physical attractiveness stereotypes and service quality in customer-server encounters. The Service Industries Journal, United Kingdom, v. 29, n. 8, p. 1.093-1.104, 2009.

MATTSSON, J. Improving Service Quality in Person-toPerson Encounters: Integrating Findings from a Multidisciplinary Review. The Service Industries Journal, United Kingdom, v. 14, n. 1, p. 45-61, 1994.

MELS, Gerhard; BOSHOFF, Christo; NEL, Deon. The Dimensions of Service Quality: The Original European Perspective Revisited. The Service Industries Journal, United Kingdom, v. 17, n. 1, p. 173-189, 1997.

NADIRI, H.; KANDAMPULLYB, J.; HUSSAIN, K. Zone of tolerance for banks: a diagnostic model of service quality. The Service Industries Journal, United Kingdom, v. 29, n. 11, p. 1.547-1.564, 2009.

NAVARRO, M. M.; IGLESIAS, M. P.; TORRES, P. R. A new management element for universities: satisfaction with the offered courses. International Journal of Educational Management, USA, v. 19, n. 6, p. 505-526, 2005.

NIELSEN, J. F; HOST, V. The Paht to service encounter Performance in public and private "bureaucracies". The Service Industries Journal, United Kingdom, v. 20, n. 1, p. 40-60, 2000.

OAKLAND, J. S. Systematic Quality Management in Banking. The Service Industries Journal, United Kingdom, v. 6, n. 2, p. 193-204, 1986.

OBEROI, U.; HALES, C. Assessing the Quality of the Conference Hotel Service Product: Towards an Empirically Based Model. The Service Industries Journal, United Kingdom, v. 10, n. 4, p. 700-721,1990.

OLORUNNIWO, F; HSU, M. K.; UDO, G. J.

Operationalizing the Service Quality Construct: The Case of the Lodging Industry. Services Marketing Quarterly, London, UK, v. 24, n. 4, p. 57-76, 2003. 
OLSON, E.; SLATER, S.; HULT, G. The performance implications of fit between Business Strategy,

Organizational Structure, Marketing and Strategic

Behavior. Journal of Marketing, Chicago: AMA, v. 69, n. 3, p. 49-65, 2005.

OZDEMIR, V. E.; HEWETT, K. The Effect of Collectivism on the Importance of Relationship Quality and Service Quality for Behavioral Intentions: A Cross-National and Cross-Contextual Analysis. Journal of International Marketing, Chicago, United States of America: AMA, v. 18, n. 1, p. 41-62, 2010.

PARASURAMAN, A.; ZEITHAML, V. A.; BERRY, L. L. Alternative Scales for Measuring Service Quality: A Comparative Assessment Based on Psychometric and Diagnostic Criteria," Journal of Retailing, Cambridge, MA, USA, n. 70, p. 201-230, 1994.

PARASURAMAN, A.; ZEITHAML, V. A; BERRY, L. L.

Reassessment of expectations as a comparison standard in measuring service quality: Implications for future research. Journal of Marketing, Cambridge, MA, v. 58, p. 111124, 1994.

PARASURAMAN, A.; ZEITHAML, V. A.; BERRY, L. L. Refinement and reassessment of the Servqual Scale, Journal of Retailing, Cambridge, MA, USA, v. 67, n. 4, p. 420-450, 1991.

PARASURAMAN, A.; ZEITHAML, V. A.; BERRY, L. L. A conceptual model of service quality and its implications for further research. Journal of Marketing, Chicago: AMA, v. 49, p. 41-50, 1985.

PARASURAMAN, A.; ZEITHAML; V. A.; BERRY, L .L. SERVQUAL: a multiple-item scale for measuring consumer perceptions of service quality. Journal of Retailing, Cambridge, MA, USA, v. 64, n. 1, p. 12-37, 1988.

PEIRÓ, J. M.; MARTINEZ-TUR, V.; RAMOS, J. Employees' Overestimation of Functional and Relational Service Quality: A Gap Analysis. The Service

Industries Journal, United Kingdom, v. 25, n. 6, p. 773$788,2005$.

PINAR, M.; ESER, Z.; STRASSER, S. Using Mystery Shopping to Compare the Service Quality of Banks in Turkey. Service Marketing Quarterly, London, UK, v. 31, n. 1, p. 1-26, 2010.
PINHEIRO, Lena Vânia Ribeiro. Lei de Bradford: uma reformulação conceitual. Ciência da Informação, Brasília, v. 12, n. 2, p. 59-80, jul.-dez. 1983.

PITT, L. F; WATSON, R. T; KAVAN, C. Measuring Information Systems Service Quality: Concerns for a Complete Canvas. MIS Quarterly, University of Minnesota, USA, v. 21, n. 2, p. 209-221, 1997.

PITT, L. F; WATSON, R. T; KAVAN, C. B. Service Quality: A Measure of Information Systems Effectiveness. MIS Quarterly, University of Minnesota, USA, v. 22, n. 1, p. 173-187, 1995.

PITT, L. F; JEANTROUT, B. Management of Customer Expectations in Service Firms: A Study and a "Checklist". The Service Industries Journal, United Kingdom, v. 14, n. 2, p. 170-189, 1994.

PITT, L.; MORRIS, M. H.; OOSTHUIZEN, P. Expectations of Service Quality as an Industrial Market Segmentation Variable. The Service Industries Journal, United Kingdom, v. 16, n. 1, p. 1-9, 1996.

PODNAR, K.; GOLOB, U. Friendly flexible working practices within the internal marketing framework: a service perspective. The Service Industries Journal, United Kingdom, v. 30, n. 11, p. 2.081-2.092, 2010.

RAHMAN, Z. Superior Service Quality in Indian Cellular Telecommunication Industry: A Source of Sustainable Competitive Advantage in an Emerging Economy.

Service Marketing Quarterly, London, UK, v. 27, n. 4, p. 115-139, 2006.

RHEE, S. K.; RHA, J. Y. Public service quality and customer satisfaction: exploring the attributes of service quality in the public sector. The Service Industries Journal, United Kingdom, v. 29, n. 11, p. 1.491-1.512, 2009.

RHYNE, D. M. The Impact of Demand Management on Service System Performance. The Service Industries Journal, United Kingdom, v. 8, n. 4, p. 446-458, 1988.

RIESENBERGER, J. R. Executive Insights: Knowledge - The Source of Sustainable Competitive Advantage. Journal of International Marketing, United States of America: AMA,v. 6, n. 3, p. 94-107, 1998. 
ROSS, G. F. Interpersonal Stress Reactions and Service Quality Responses among Hospitality Industry Employees. The Service Industries Journal, United Kingdom, v. 15, n. 3, p. 314-331, 1995.

SALEH, F; RYAN, C. Analysing Service Quality in the Hospitality Industry Using the SERVQUAL Model. The Service Industries Journal, United Kingdom, v. 11, n. 3, p. 324-345, 1991.

SANCHEZ-PEREZ, M et al. Service Quality in Public Services as a Segmentation Variable. The Service Industries Journal, United Kingdom, v. 27, n. 4, p. 355369, 2007.

SCHLEGELMILCH, B. B.; CARMAN, J. M.; MOORE, S. A. Choice and Perceived Quality of Family Practitioners in the United States and the United Kingdom. The Service Industries Journal, United Kingdom, v. 12, n. 2, p. 263284, 1992.

SHARIF, K. Relationship Between Intra- and Interorganizational Service Quality Perceptions: An Empirical Testing of Modified Service Profit Chain Model Within Kuwaiti Telecommunications Industry. Service Marketing Quarterly, London, UK, v. 30, n. 3, p. 249269, 2009.

SHEPPARD, L. Network Development and application in Health Care: A Study of Service Quality. Services

Marketing Quarterly, London, UK, v. 24, n. 3, p. 43-61, 2003.

SHREDNICK, H.; SHUTT, R.; WEISS, M. Empowerment: Key to IS World-Class Quality. MIS Quarterly, University of Minnesota, USA, v. 16, n. 4, p. 491-504, 1992.

SHUKLA, P. Effects of Perceived Sacrifice, Quality, Value, and Satisfaction on Behavioral Intentions in the Service Environment. Service Marketing Quarterly, London, UK, v. 31, n. 4, p. 466-484, 2010.

SICHTMANN, C.; SELASINSKY, M. V.; DIAMANTOPOULOS, A. Service Quality and Export Performance of Business-to-Business Service Providers: The Role of Service Employee- and Customer-Oriented Quality Control Initiatives. Journal of International Marketing, United States of America: AMA, v. 19, n. 1, p. 1-22, 2011.
SLATER, S.; OLSON, E. Type of strategy and performance: The influence of sales force management. Journal of Strategic Management, University of East Anglia, UK, 2000.

SMITH, Anne M. The Impact of Scale Characteristics on the Dimensionality of the Service Quality Construct. The Service Industries Journal, United Kingdom, v. 20, n. 3, p. 167-190, 2000.

SMITH, D. J. Computer Simulation Applications in Service Operations: A Case Study from the Leisure Industry. The Service Industries Journal, United Kingdom, v. 14, n. 3, p. 395-408, 1994.

SNELL, L.; WHITE, L. A Sociocognitive Approach to Service Quality and Adherence Amongst Elderly Patients: A Pilot Study. Health Marketing Quarterly, London, UK, v. 28, n. 2, p. 99-115, 2011.

STEINBART, P. J.; NATH, R. Problems and Issues in the Management of International Data Communications Networks: The Experiences of American Companies. MIS Quarterly, University of Minnesota, USA, v. 16, n. 1, p. 55-76, 1992.

SU, H. Y.; LIN, Y. Enhancing Knowledge-Based Service Quality: A Knowledge Management Perspective. The Service Industries Journal, United Kingdom, v. 26, n. 7, p. 787-800, 2006.

TEAS, R. K. Expectations, performance evaluation, and customers perceptions of quality. Journal of Marketing, Chicago, AMA, v. 57, n. 4, p. 18-34, 1993.

WANG, C. Y. Investigating antecedents of consumers' recommend intentions and the moderating effect of switching barriers. The Service Industries Journal, United Kingdom, v. 29, n. 9, p. 1.231-1.241, 2009.

WANG, M, L. Does organisational support promote citizenship? The moderating role of market-focused HRM.

The Service Industries Journal, United Kingdom, v. 30, n. 7, p. 1.077-1.095, 2010.

WATSON, R. T.; PITT, L. F.; KAVAN, C. B. Measuring Information Systems Service Quality: Lessons From Two Longitudinal Case Studies. MIS Quarterly, University of Minnesota, USA, v. 22, n. 1, p. 61-79, 1998. 
WEBB, D. Understanding Customer Role and its Importance in the Formation of Service Quality Expectations. The Service Industries Journal, United Kingdom, v. 20, n. 1, p. 89-101, 2000.

WHITE, C. J. Towards an Understanding of the Relationship between Mood, Emotions, Service Quality and Customer Loyalty Intentions. The Service

Industries Journal, United Kingdom, v. 26, n. 8, p. 837847, 2006.

WILLBORN, W. Quality Assurance Audits and Hotel Management. The Service Industries Journal, United Kingdom, v. 6, n. 3, p. 293-308, 1986.

WILLIAMS, Christine S.; SAUNDERS, Mark N. K. Developing the Service Template Process: From Measurement to Agendas for Improvement. The Service Industries Journal, United Kingdom, v. 26, n. 5, p. 581595, 2006.

WITT, C. A.; STEWART, H. M. Solicitors and Customer Care. The Service Industries Journal, United Kingdom, v. 16, n. 1, p. 21-34, 1996.

WU, H. L.; LIU, C. Y.; HSU, W. H. An integrative model of customers' perceptions of health care services in Taiwan. The Service Industries Journal, United Kingdom, v. 28, n. 9, p. 1.307-1.319, 2008.

YANG, C. C. A Systems Approach to Service Development in a Concurrent Engineering Environment. The Service Industries Journal, United Kingdom, v. 27, n. 5, p. 635-652, 2007.

YANG, C. C. et al. Methods for determining areas for improvement based on the design of customer surveys.

The Service Industries Journal, United Kingdom, v. 29, n. 2, p. 143-154, 2009.

YELKUR, R.; CHAKRABARTY, S. Gender Differences in Service Quality Expectations in the Fast Food Industry.

Service Marketing Quarterly, London, UK, v. 27, n. 4, p. 141-151, 2006.

YU, C. S. Assessing and differentiate the quality of Internet-based services: a case of online banking services in Taiwan. The Service Industries Journal, United Kingdom, v. 28, n. 5, p. 581-602, 2008.
ZEITHAML, V.; PARSURAMAN, A. BERRY, L. L.

Delivering Service Quality: Balancing Customer Perceptions and expectations. New York: The Free Press, 1991.

ZEITHAML, V. A.; BERRY, L. L.; PARASURAMAN, A. Communication and control processes in the delivery of service quality. Journal of Marketing, Chicago: AMA, v. 52, p. 35-48. 1988.

ZEITHAML, V. A.; BERRY, L. L.; PARASURAMAN, A. The behavioural consequences of service quality. Journal of Marketing, Chicago: AMA, v. 60, n. 4, p. 31-46. 1996.

ZEITHAML, V. A.; BERRY, L. L; PARASURAMAN, A. The nature and determinants of customer expectations of service. Journal of the Academy of Marketing Science, Louisiana, USA, n. 21, p. 1-12. 1993. 